\title{
Candidemia in the Neonatal Intensive Care Unit: A Retrospective, Observational Survey and Analysis of Literature Data
}

\author{
Giuseppina Caggiano, ${ }^{1}$ Grazia Lovero, ${ }^{1}$ Osvalda De Giglio, ${ }^{1}$ Giovanna Barbuti, ${ }^{2}$ \\ Osvaldo Montagna, ${ }^{3}$ Nicola Laforgia, ${ }^{4}$ and Maria Teresa Montagna ${ }^{1}$ \\ ${ }^{1}$ Department of Biomedical Science and Human Oncology, Hygiene Section, University of Bari "Aldo Moro", Bari, Italy \\ ${ }^{2}$ Department of Biomedical Science and Human Oncology, General Pathology Section, University of Bari "Aldo Moro”, Bari, Italy \\ ${ }^{3}$ Neonatology and NICU Section, Azienda Ospedaliero-Universitaria Policlinico of Bari, Bari, Italy \\ ${ }^{4}$ Department of Biomedical Science and Human Oncology, Neonatology and NICU Section, University of Bari "Aldo Moro", Bari, Italy
}

Correspondence should be addressed to Giuseppina Caggiano; giuseppina.caggiano@uniba.it

Received 16 March 2017; Accepted 18 June 2017; Published 13 August 2017

Academic Editor: Stanley Brul

Copyright ( 2017 Giuseppina Caggiano et al. This is an open access article distributed under the Creative Commons Attribution License, which permits unrestricted use, distribution, and reproduction in any medium, provided the original work is properly cited.

\begin{abstract}
We evaluated the epidemiology of Candida bloodstream infections in the neonatal intensive care unit (NICU) of an Italian university hospital during a 9-year period as a means of quantifying the burden of infection and identifying emerging trends. Clinical data were searched for in the microbiological laboratory database. For comparative purposes, we performed a review of NICU candidemia. Forty-one candidemia cases were reviewed (overall incidence, 3.0 per 100 admissions). Candida parapsilosis sensu stricto (58.5\%) and C. albicans (34.1\%) were the most common species recovered. A variable drift through years was observed; in 2015, 75\% of the cases were caused by non-albicans species. The duration of NICU hospitalization of patients with non-albicans was significantly longer than in those with C. albicans (median days, 10 versus 12). Patients with non-albicans species were more likely to have parenteral nutrition than those with C. albicans (96.3\% versus 71.4\%). Candida albicans was the dominant species in Europe and America (median, 55\% and 60\%; resp.); non-albicans species predominate in Asia (75\%). Significant geographic variation is evident among cases of candidemia in different parts of the world, recognizing the importance of epidemiological data to facilitate the treatment.
\end{abstract}

\section{Introduction}

Although blood stream infection (BSI) due to Candida species (spp.) in the neonatal intensive care unit (NICU) is less frequent than that due to Gram-positive or Gram-negative bacteria, it has higher morbidity and mortality rates. In particular, among newborns with a birth weight $<1000 \mathrm{~g}$, $4-8 \%$ will develop candidemia, which has a $30 \%$ mortality in this group of patients [1]. Newborns who survive frequently have long-term neurological impairment, including cerebral palsy, blindness, hearing impairment, cognitive deficits, and periventricular leukomalacia [2]. Risk factors for neonatal candidemia include prematurity, use of central venous lines, endotracheal tubes, parenteral nutrition, broad-spectrum antibiotic administration (especially third-generation cephalosporins), prolonged hospitalization, abdominal surgery, exposure to $\mathrm{H} 2$ blockers, and Candida colonization. Although Candida albicans is the most prevalent yeast pathogen, BSIs caused by Candida non-albicans, particularly Candida parapsilosis complex and Candida glabrata complex, have increased in recent years $[2,3]$.

This study aimed (i) to determine the epidemiology of Candida BSIs in the NICU of an Italian university hospital during 9 years of observation; (ii) to analyze the trend in species distribution; and (iii) to examine in vitro susceptibility to common antifungal drugs. Furthermore, for comparative purposes, a systematic review of studies concerning the distribution of Candida spp. causing candidemia in NICU patients is presented. 


\section{Materials and Methods}

2.1. Study Design. A retrospective, observational survey of all consecutive cases of candidemia was conducted at the NICU (capacity of 8 beds; level III) of a university hospital in Southern Italy, from January 1, 2007, to December 31, 2015. The number of annual admissions ranged from 135 to 169 , with no significant variation during the period of study. All of the neonates who had at least one positive blood culture for Candida spp. and signs or symptoms of infection were considered in this study. Only the first episode of candidemia was reported for patients with recurrent or subsequent episodes. Clinical data were searched for in the microbiological laboratory database and included sex, gestational age, birth weight, and predisposing risk factors for Candida BSI (i.e., intravascular devices, prolonged antibiotics, administration of total parental nutrition, and prolonged hospitalization).

2.2. Definitions. Extremely low birth weight (ELBW) infants were defined as those with a birth weight $\leq 1000 \mathrm{~g}$, very low birth weight (VLBW) infants were those with a birth weight $<1500 \mathrm{~g}$, and low birth weight infants were those with a birth weight $<2500 \mathrm{~g}$. Prolonged antibiotic use was defined as $>14$ days of continuous administration. Late-onset sepsis (LOS) was defined as infection occurring for $>48 \mathrm{~h}$ of life. Candidemia was considered as probably catheter-related when semiquantitative culture of the catheter tip yielded $>15$ colony-forming units of Candida.

2.3. Laboratory Procedures. Blood cultures were performed using a lysis-centrifugation system (Isolator; DuPont Co., Wilmington, DE, USA). The samples were cultured on two plates of Sabouraud dextrose agar with $0.05 \%$ chloramphenicol (BioRad, Marnes-la-Coquette, France) and then incubated at $36^{\circ} \mathrm{C}( \pm 1)$ and $28^{\circ} \mathrm{C}( \pm 1)$. The samples were examined daily for 10 days. The isolates were identified using standard procedures (morphology on cornmeal agar plates, germ-tube production in serum, and ability to grow at $37^{\circ} \mathrm{C}$ and $42^{\circ} \mathrm{C}$ ) and biochemical analysis using two methods, the Vitek 2 system and ID 32C panels (Bio-Merieux, Rome, Italy), to obtain accurate results. All strains were frozen at $-70^{\circ} \mathrm{C}$ until further investigations [4]. Candida parapsilosis complex genotyping was performed by PCR amplification as reported previously $[5,6]$.

Antifungal susceptibility tests to five antifungal drugs (anidulafungin, fluconazole, caspofungin, micafungin, and amphotericin B) were performed for all Candida spp., using the Sensititre YeastOne technique (SYO-09 panel; Trek Diagnostic Systems, Ltd., East Grinstead, England).

The susceptibility values were interpreted taking into account the species-specific clinical breakpoints (CBPs) suggested by the Clinical Laboratory Standards Institute (CLSI) subcommittee for the most common species of Candida [7]. The epidemiological cut-off values were used to define wildtype and non-wild-type isolates if no CBPs were available from the CLSI $[8,9]$. Minimum inhibitory concentration (MIC) data are presented as MIC $_{50}$ (MIC causing inhibition of $50 \%$ of isolates) and $\mathrm{MIC}_{90}$ (MIC causing inhibition of $90 \%$ of isolates).
2.4. Statistical Analysis. The Shapiro-Wilk test was used to test the normal distribution of data. Non-normally distributed data are expressed as median and interquartile range (IQR) and were compared using the Mann-Whitney $U$ test. Categorical data are expressed as number and percentage and were compared using $\chi^{2}$ or Fisher's exact test. All $p$ values are two-tailed, and statistical significance was defined as $p<0.05$ (Social Sciences (SPSS) software 10 for Mac OS X; SPSS Inc., Chicago, IL, USA).

2.5. Literature Review. A review of full-text articles that were published in English from January 2000 to February 2015 was performed. The MEDLINE database was used for the bibliographic research, using the following key words: "neonatal candidemia", "candidemia neonatal intensive care unit", "Candida neonatal intensive care unit, and "NICU candidemia". Additionally, the bibliographies of the selected articles were reviewed for relevant publications.

The exclusion criteria were as follows: articles that reported a period of study prior to 2000; letters, randomized, controlled trials; and studies that reported a total number of Candida BSIs less than five. The following data were collected from each selected study: geographic location, year of publication, study period, type of study, incidence, influencing factors candidemia, total number of isolated Candida spp., and relative proportion of each of the Candida spp.

\section{Results}

3.1. Analysis of Cases in the NICU. A total of 41 infants with Candida infection were reviewed. The overall incidence of candidemia was 3.0 per 100 NICU admissions (range, 2.2-3.0). The male: female ratio was $1.6: 1$. The cohort had a median gestational age of 30 weeks (29-31 weeks) and a median birth weight of $1110 \mathrm{~g}$ (900-1345 g). The majority of candidemia episodes occurred in VLBW infants (56.1\%). The median duration of the total hospital stay was 11 days (8-14 days). Candidemia was catheter-related in 23 cases (56.1\%). All Candida infections were classified as LOS. At the moment of candidemia, only ELBW infants were receiving antifungal prophylaxis with fluconazole ( $3 \mathrm{mg} / \mathrm{kg} /$ day).

Candida parapsilosis sensu stricto was isolated with the highest frequency (58.5\%), followed by C. albicans (34.1\%), C. glabrata complex, C. guilliermondii, and C. orthopsilosis $(2.4 \%$ for each). Therefore, $65.9 \%$ of candidemia episodes were caused by Candida non-albicans. With regard to the temporal trend of C. albicans and Candida non-albicans, a variable drift from 2007-2015 was observed, with a considerable percentage (75\%) increase in non-albicans species in 2015 (Figure 1). Predisposing factors associated with C. albicans and non-albicans are listed in Table 1. The duration of NICU hospitalization of patients with $C$. non-albicans was significantly longer than that in those with C. albicans (median days, 10 [7.5-12] versus 12 [10-15], $p=0.045$ ). Patients with $C$. nonalbicans were more likely to have parenteral nutrition than those with $C$. albicans $(96.3 \%$ versus $71.4 \%, p=0.039$ ).

Results of antifungal susceptibility are shown in Table 2. All of the strains were sensitive to tested drugs. Overall, the 
TABLE 1: Clinical characteristics of the patients with candidemia by species.

\begin{tabular}{lccc}
\hline Characteristics & Candida albicans $(n=14)$ & Candida non-albicans $(n=27)$ & $p$ value \\
\hline Low gestational age $\leq 32 \mathrm{wk}, n(\%)$ & $11(78.6)$ & $25(92.6)$ & 0.317 \\
Gestational age $^{\ddagger}$ & $31(29.5-31.5)$ & $30(29-31)$ & 0.193 \\
Birth weight $\leq 1500 \mathrm{~g}, n(\%)$ & $11(78.6)$ & $1200(900-1380)$ & 0.317 \\
Birth weight $(\mathrm{g})^{\ddagger}$ & $1200(1013-1625)$ & $27(100)$ & 0.573 \\
Stay in NICU $\leq 7$ days, $n(\%)$ & $12(85.7)$ & $12(10-15)$ & 0.111 \\
Length of stay before candidemia (days) & $10(7.5-12)$ & $27(100)$ & $\mathbf{0 . 0 4 5}$ \\
Presence of CVC, $n(\%)$ & $13(92.8)$ & $26(96.3)$ & 0.342 \\
TPN, $n(\%)$ & $10(71.4)$ & $26(96.3)$ & $\mathbf{0 . 0 3 9}$ \\
Mechanical ventilation, $n(\%)$ & $11(78.6)$ & $24(92.3)$ & 0.107 \\
Prolonged antibiotic therapy, $n(\%)$ & $12(85.7)$ & 1.000 \\
\hline
\end{tabular}

${ }^{\ddagger}$ Median (interquartile range). CVC: central venous catheter; TPN: total parenteral nutrition. Bold values are significant.

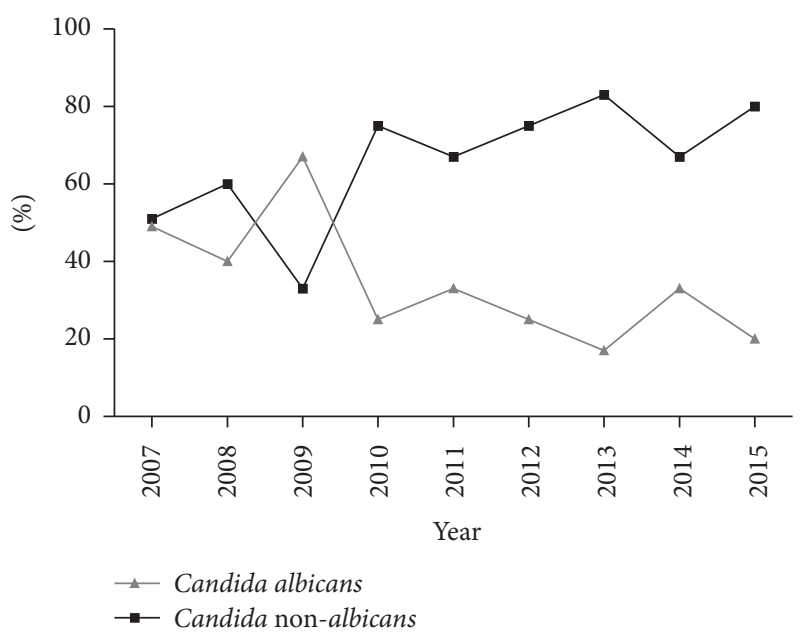

Figure 1: Temporal trend of Candida albicans and Candida nonalbicans during a 9-year period.

$\mathrm{MIC}_{50} / \mathrm{MIC}_{90}$ values $(\mathrm{mg} / \mathrm{L})$ were as follows: amphotericin $\mathrm{B}$, $0.25 / 0.5$; anidulafungin, $1 / 2$; caspofungin, $0.25 / 0.5$; fluconazole, $0.5 / 2$; and micafungin, $1 / 1$.

3.2. Literature Review. A total of 45 articles were selected (Tables 3 and 4). Thirty-two studies reported data from a single hospital and 27 were retrospective studies. Seventeen studies were conducted in Asia, 13 in Europe, 11 in North and South America, and 2 in South Africa. Finally, one cohort was carried out in Australia.

The distribution of Candida spp. varied according to the different geographical areas. Candida albicans was the dominant species in Europe with proportions ranging from 47 to $100 \%[10,11,13,14,16,18,19,22,23,53]$ and in North and South America with proportions ranging from 40 to $69.2 \%$ [24-31, 33, 34]. Candida non-albicans species were predominant in Asia [36-40, 42, 43, 45, 47, 48], with proportions ranging from 25 to $92 \%$, with a median of $75 \%$

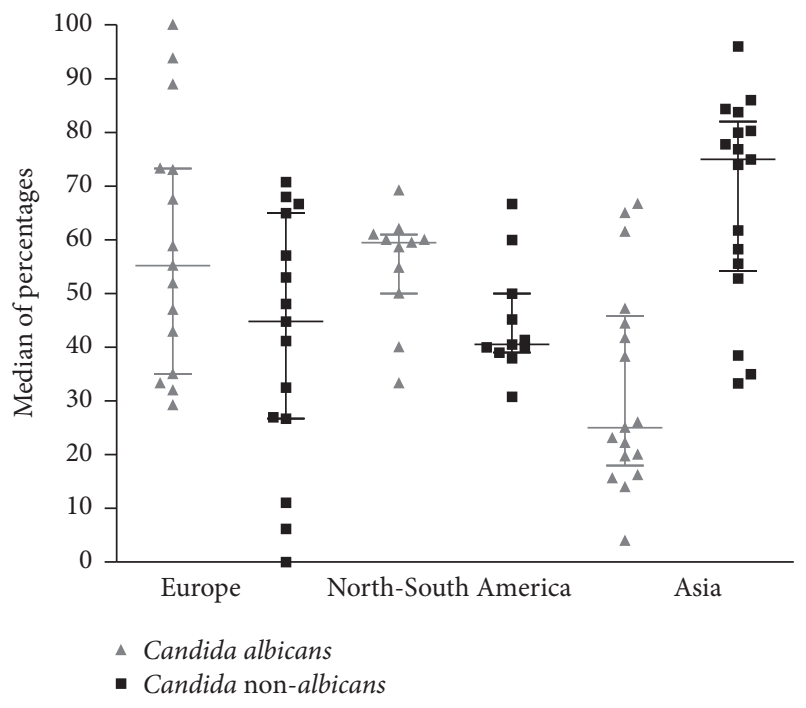

FIGURE 2: Distribution of Candida spp. according to the different geographical areas.

(Figure 2). In Australia, C. albicans and C. non-albicans were equally distributed ( $42 \%$ and $43 \%$, resp.) [52].

For C. non-albicans, the three most prevalent species were C. parapsilosis complex, C. glabrata complex, and C. tropicalis. Generally, C. parapsilosis complex was the second most common pathogen (range, 6.2-77.8\%). C. parapsilosis complex was the predominant species in some studies from Europe $[12,15,17,20,21]$ and Asia [37, 40, 42, 45, 48]. The highest proportions of $C$. glabrata complex were reported in studies that were conducted in the central part of India (range, 22.2-44.4\%), while the lowest proportions were observed in European countries (range, 2.5-5.9\%). No cases due to C. glabrata complex were reported in South America. The highest frequency of $C$. tropicalis was found in South India (36.7-92\%), followed by studies from South America (11.2-13.3\%) and South Africa (8.8\%). The lowest frequencies were observed in Europe (3.7-5\%) and Australia (2\%). There were no reports of $C$. tropicalis in North America. 


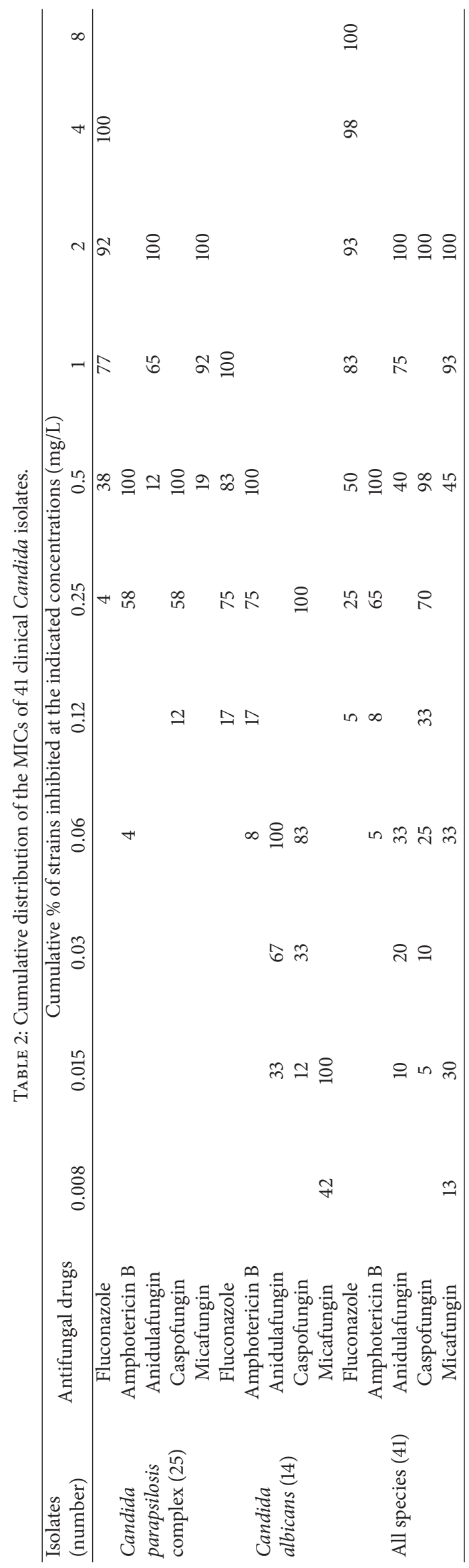




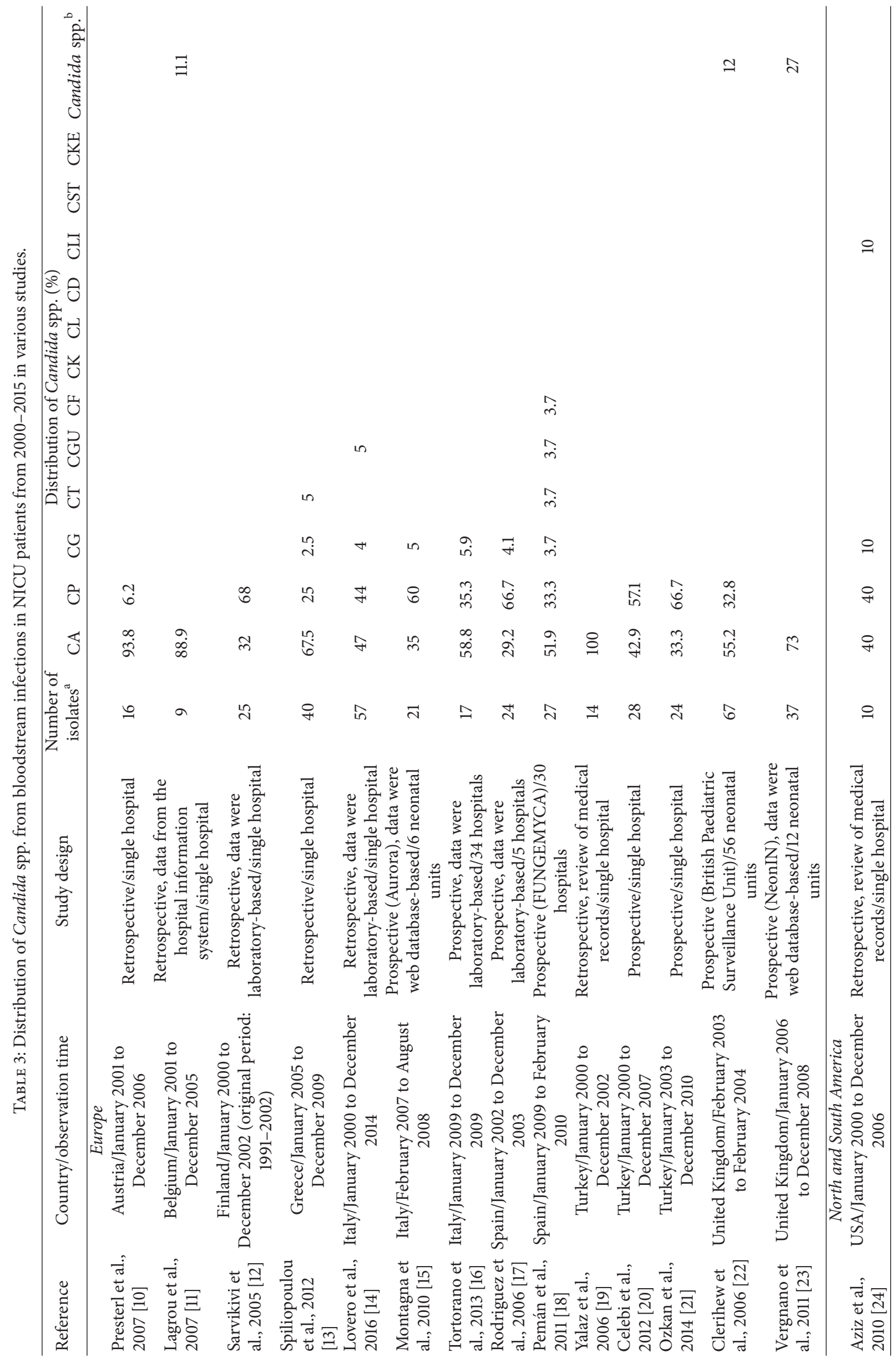




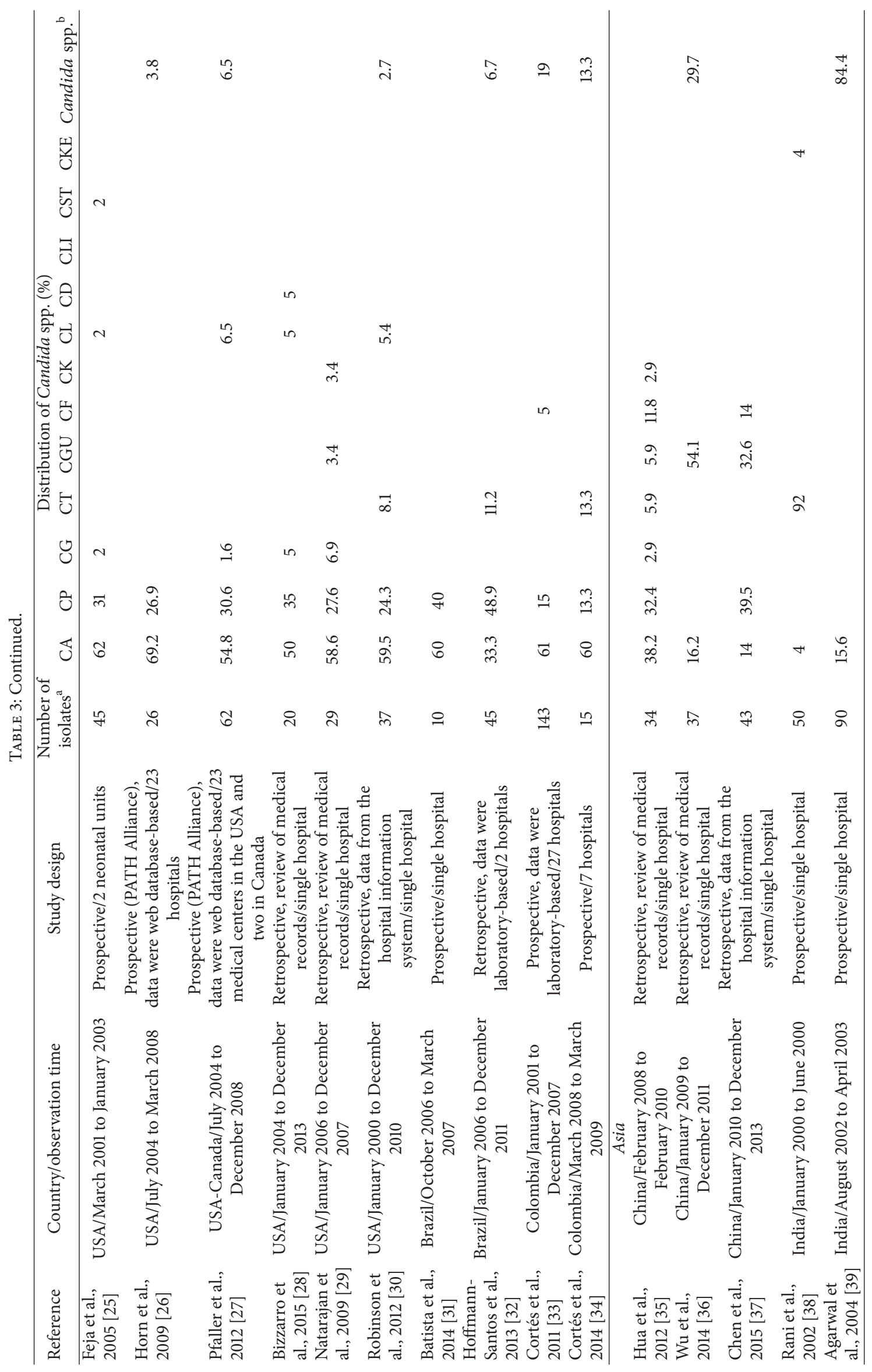




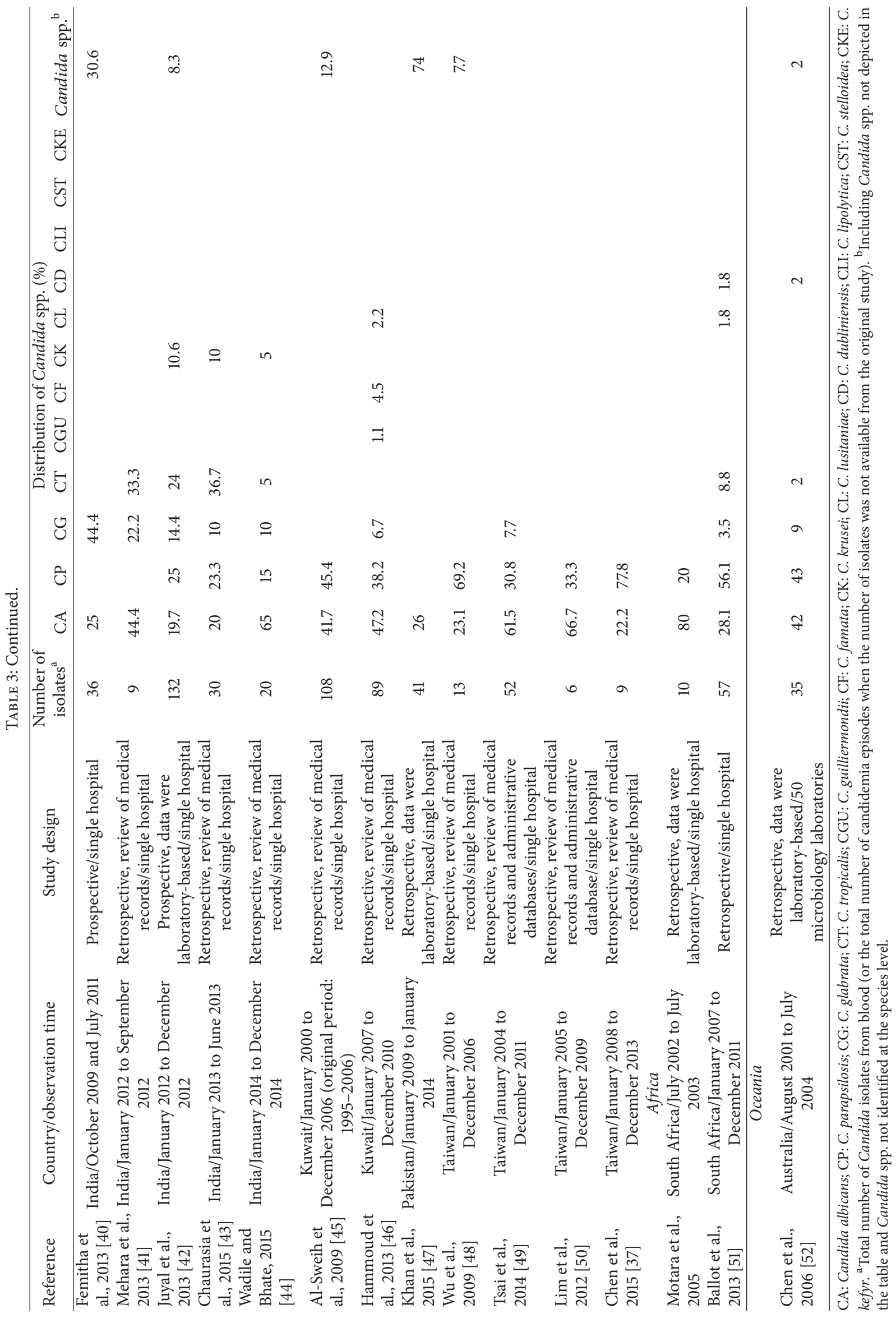


TABLE 4: Main candidemia finding in the NICU as reported in various studies.

Reference

Lagrou et al., 2007 [11]

Sarvikivi et al., 2005 [12]

Spiliopoulou et al., 2012

[13]

Lovero et al., 2016 [14]

Montagna et al., 2010

[15]

Rodriguez et al., 2006

[17]

Pemán et al., 2011 [18]

Yalaz et al., 2006 [19]

Celebi et al., 2012 [20]

Ozkan et al., 2014 [21]

Clerihew et al., 2006 [22

Vergnano et al., 2011 [23]

Aziz et al., 2010 [24]

Feja et al., 2005 [25]

Bizzarro et al., 2015 [28]

Natarajan et al., 2009

[29]

Robinson et al., 2012 [30]

Batista et al., 2014 [31]

Hua et al., 2012 [35]

Wu et al., 2014 [36]

Chen et al., 2015 [37]

Rani et al., 2002 [38]

Agarwal et al., 2004 [39]

Femitha et al., 2013 [40]

Mehara et al., 2013 [41]

Juyal et al., 2013 [42]

Chaurasia et al., 2015

[43]

Al-Sweih et al., 2009 [45]

Hammoud et al., 2013

[46]

Wu et al., 2009 [48]
Main candidemia finding in the NICU

Annual incidence: 0.30 episodes per 10,000 patient-days.

Fluconazole prophylaxis contributed to the emergence of C. parapsilosis with decreased susceptibility to fluconazole.

Candidemia incidence decreased. C. albicans was most frequently isolated from ELBW infants. Mortality (35.7\%) was associated with low gestational age and low birth weight.

Incidence rate of Candida non-albicans increased from 46\% in 2000-2004 to 71\% in 2010-2014.

Overall incidence: 1.3 per 100 NICU discharges. The incidence in ELBW infants was $4.3 \%$ versus $0.2 \%$ in LBW infants.

Annual incidence: 1.1 per 100 NICU discharges and 1.08 per 1000 patient-days. Low mortality (21\%) rate may have been caused by a high prevalence of $C$. parapsilosis fungemia.

C. albicans was more common in the NICU setting than in the pediatric ICU.

Candidemia markedly increased in 2002 compared with previous years. A significant association was found between Candida infection and the duration of antibiotic therapy.

Overall incidence: 11.5 per 1000 NICU admissions. The mortality rate was $42.8 \%$.

Gram-positive sepsis (67.6\%) was more common than Gram-negative bacteremia (16.6\%) and candidemia (15.8\%). Candida spp. caused LOS (58.3\%), VLOS (41,7\%), and no EOS sepsis.

C. parapsilosis was associated with fewer deep-seated infections than C. albicans, but mortality was similar.

A decrease in candidemia was observed: $1.8 \%$ in $2006,1.2 \%$ in 2007 , and $1.3 \%$ in 2008. Candida spp. were more common in LOS (97\%) than in EOS (3\%) sepsis.

Fluconazole prophylactic administration to ELBW infants was associated with a decreased rate of candidemia.

Overall incidence: 1.6 per 100 NICU discharges. Catheter use, previous bacterial sepsis, and GI pathology were significantly associated with candidemia.

Candida spp. were more common in LOS than in EOS sepsis.

Candidemia refractory to conventional antifungals was associated with prolonged antibiotic use and Candida non-albicans infection.

Overall incidence: 0.45 per 100 NICU discharges. An increased time between blood culture draw and initial antifungal therapy was associated with an increased incidence of persistent candidemia.

Oral colonization should be considered as a risk factor for candidemia.

Patients with C. parapsilosis had a significantly longer hospital stay than those with C. albicans sepsis.

C. guilliermondii was associated with preterm infants and with low birth weight.

Fluconazole prophylaxis alone was not efficacious; it had to be combined with reinforcement of management and supervision of hand hygiene to effectively prevent invasive candidiasis.

Candida non-albicans accounted for $96 \%$ of the cases of neonatal candidemia.

Overall incidence: 77 per 1000 NICU discharges. Candida non-albicans is gaining importance as a cause of neonatal septicemia.

Overall incidence: 0.82 cases per 100 NICU discharges. Mortality was $44.4 \%$. Presence of candiduria was a significant riskfactor for death.

Candida spp. were more common in LOS than in EOS sepsis.

Candida non-albicans accounted for $80.30 \%$ of the cases of neonatal candidemia. The crude mortality was $34.85 \%$.

Clinical features in neonates with candida sepsis were nonspecific. A common laboratory feature was thrombocytopenia.

Overall incidence: 4 per 100 NICU discharges.

C. albicans was the most prevalent species in nonpersistent candidemia. C. parapsilosis was more common among infants with persistent candidemia. Persistent candidemia was associated with an increased risk of mortality.

The most common causative microorganisms of LOS sepsis were CONS and Candida spp. C. parapsilosis was associated with a high mortality rate. 
TABLE 4: Continued.

\begin{tabular}{ll}
\hline Reference & Main candidemia finding in the NICU \\
\hline Tsai et al., 2014 [49] & $\begin{array}{l}\text { Candidemia had a significantly higher rate of infectious complications, persistent bloodstream infection, and } \\
\text { sepsis-attributable mortality than Gram-negative and Gram-positive bacteremia. } \\
\text { Sepsis by Gram-negative bacteria or Candida spp. presented with more severe clinical symptoms and was } \\
\text { associated with a higher mortality rate compared with that by Gram-positive bacteria. }\end{array}$ \\
Lim et al., 2012 [50] & $\begin{array}{l}\text { Decrease incidence of candidemia during the study period. } \\
\text { Increased incidence of Candida non-albicans during the study period. }\end{array}$ \\
\hline
\end{tabular}

CONS: coagulase-negative staphylococci; ELBW: extremely low birth weight; VLBW: very low birth weight; GI: gastrointestinal; EOS: early-onset sepsis; LOS: late-onset sepsis; VLOS: very late-onset sepsis; NICU: neonatal intensive care unit; ICU: intensive care unit.

\section{Discussion}

This study aimed to describe the epidemiology and drug susceptibility of Candida isolates causing candidemia in a NICU of an Italian university hospital over 9 years. Our survey showed that candidemia is a common problem among critically ill neonates, with an overall incidence of $3 \%$. This finding is higher than data reported in a literature review from Europe $(1.1-1.3 \%)[15,17]$ and the North and South America (0.5-1.6\%) [25, 30], but lower than that reported in Asia (4-7.7\%) [39, 45]. This variability may reflect differences in health care practices among countries, as well as the study design adopted, including differences in the examined population.

VLBW infants are known to be at a high risk of candidemia because of more aggressive and invasive therapies, such as indwelling central lines, mechanical ventilation, parenteral hyperalimentation, and longer hospital stay [1$3]$. The majority of infected neonates have a gestational age at birth of 30 weeks or earlier and birth weight is $\leq 1500 \mathrm{~g}$ (87.8\%, each one). Intravenous catheters are risk factors for Candida BSI in critically ill infants. We found that all patients had intravenous catheter placement and that candidemia was catheter-related in $56.1 \%$ of cases. This finding is not surprising because Candida spp. can adhere to platelets and fibrinogen on the surface of catheters and form biofilms that may become a reservoir for systemic spread [1-3].

In our systematic review, we found that only four species (C. albicans, C. parapsilosis complex, C. tropicalis, and C. glabrata complex) accounted for $95.4 \%$ of cases of candidemia. However, the ranking of these four species was variable. Generally, C. albicans was the predominant isolated spp. in Europe [10,11, 13, 14, 16, 18, 19, 22, 23, 53] and North and South America [24-31, 33, 34]. However, non-albicans species were predominant in Asia [36-40, 42, 43, 45, 47, 48].

Moreover, data regarding changes in the relative frequencies of isolated Candida spp. showed a shift toward Candida non-albicans, with a frequency higher than $50 \%$ in some NICUs. This, in part, is attributed to the increased use of azole prophylaxis and therapy [12]. However, in a recent study, where fluconazole was rarely used for prophylaxis and therapy, a high incidence of non-albicans $(60.8 \%$ of all candidemia episodes) was found [20]. Similarly, our study showed a higher percentage of $C$. non-albicans $(66 \%)$ than C. albicans and a variable drift through 9 years. In 2015, 75\% of the cases were caused by non-albicans species.
In our study, appearance of C. parapsilosis complex as the predominant fungal pathogen (61\% of all isolates) was consistent with the pattern seen in some hospitals in Europe, Asia, and Africa [12, 15, 17, 20, 37, 42, 45, 48, 51].

Main risk factors for $C$. parapsilosis complex infection were the presence of indwelling vascular catheters and parenteral nutrition, both of which predispose to formation of biofilms. Morphogenesis from yeast cells to pseudohyphae is essential for biofilm formation and virulence in C. parapsilosis complex. Amino acids mediate cell differentiation, and this could explain the high incidence of this yeast in catheterized neonates who receive amino acid-rich parenteral nutrition solutions [54]. Our data highlights an association between parenteral nutrition and non-albicans spp. The high proportion of C. parapsilosis complex may explain this finding. Notably, we observed that NICU patients were more likely to develop C. parapsilosis sensu stricto (58.5\%) than C. orthopsilosis $(2.4 \%)$ candidemia. This finding may be explained by the greater capacity of $C$. parapsilosis sensu stricto to adhere to central lines compared with closely related species [55].

In agreement with other studies [13-15, 17, 18], none of the isolated strains showed resistance to fluconazole and amphotericin B. These are the antifungal drugs of choice that are used in prophylaxis and treatment of Candida BSI in neonates [56]. No fluconazole resistance may be related to the treatment policy in use at our hospital, where systemic antifungal prophylaxis with fluconazole was used only in ELBW infants. In neonates, fluconazole prophylaxis has been linked to the emergence of azole resistance $[12,57]$.

\section{Conclusions}

Limitations of the present study are mainly related to its retrospective nature with limited follow-up data. Although all of the data were prospectively collected, some variables could not be examined because of missing data. Furthermore, we did not have data on specific characteristics of noninfected patients in our NICU. Therefore, we were not able to riskadjust our rates to compare with incidences from other reports.

Nevertheless, this study shows that $C$. non-albicans candidemia is increasing, despite limited use of fluconazole for prophylaxis/empiric therapy in our unit. Our results also confirm that candidemia plays an important pathogenic role in NICU patients. There is a significant variation in cases of candidemia in different geographic regions, even within the 
same continent. Therefore, monitoring epidemiological data to facilitate the choice of treatment is important.

\section{Ethical Approval}

The study protocol was approved by the Ethics Committee of the Azienda Ospedaliero-Universitaria Policlinico of Bari, Italy (Application no. 1321, 2007). Registered data were managed in accordance with the Italian data protection laws (privacy law).

\section{Consent}

Written informed consent was obtained from patient parents or their legal guardians.

\section{Conflicts of Interest}

The authors declare that they have no conflicts of interest.

\section{References}

[1] D. Testoni, M. Hayashi, M. Cohen-Wolkowiez et al., "Late-onset bloodstream infections in hospitalized term infants," Pediatric Infectious Disease Journal, vol. 33, no. 9, pp. 920-923, 2014.

[2] M. S. Kelly, D. K. Benjamin, and P. B. Smith, "The epidemiology and diagnosis of invasive candidiasis among premature infants," Clinics in perinatology, vol. 42, no. 1, pp. 17-105, 2015.

[3] E. Leibovitz, "Strategies for the prevention of neonatal candidiasis," Pediatrics and Neonatology, vol. 53, no. 2, pp. 83-89, 2012.

[4] G. Caggiano, C. Coretti, N. Bartolomeo, G. Lovero, O. De Giglio, and M. T. Montagna, "Candida bloodstream infections in Italy: Changing epidemiology during 16 years of surveillance," BioMed Research International, vol. 2015, Article ID 256580, 2015.

[5] A. Tavanti, A. D. Davidson, N. A. R. Gow, M. C. J. Maiden, and F. C. Odds, "Candida orthopsilosis and Candida metapsilosis spp. nov. to replace Candida parapsilosis groups II and III," Journal of Clinical Microbiology, vol. 43, no. 1, pp. 284-292, 2005.

[6] E. Borghi, R. Sciota, R. Iatta, C. Biassoni, M. T. Montagna, and G. Morace, "Characterization of Candida parapsilosis complex strains isolated from invasive fungal infections," European Journal of Clinical Microbiology and Infectious Diseases, vol. 30, no. 11, pp. 1437-1441, 2011.

[7] Clinical and Laboratory Standards Institute, Reference method for broth dilution antifungal susceptibility testing of yeasts; fourth informational supplement, M27-S4, CLSI, Wayne, PA, USA, 2012.

[8] A. Espinel-Ingroff, M. A. Pfaller, B. Bustamante et al., "Multilaboratory study of epidemiological cutoff values for detection of resistance in eight Candida species to fluconazole, posaconazole, and voriconazole," Antimicrobial Agents and Chemotherapy, vol. 58, no. 4, pp. 2006-2012, 2014.

[9] M. A. Pfaller, M. Castanheira, S. A. Messer, and R. N. Jones, "In vitro antifungal susceptibilities of isolates of Candida spp. and Aspergillus spp. from China to nine systemically active antifungal agents: Data from the SENTRY antifungal surveillance program, 2010 through 2012," Mycoses, vol. 58, no. 4, pp. 209214, 2015.

[10] E. Presterl, F. Daxböck, W. Graninger, and B. Willinger, "Changing pattern of candidaemia 2001-2006 and use of antifungal therapy at the University Hospital of Vienna, Austria," Clinical Microbiology and Infection, vol. 13, no. 11, pp. 1072-1076, 2007.

[11] K. Lagrou, J. Verhaegen, W. E. Peetermans, T. De Rijdt, J. Maertens, and E. Van Wijngaerden, "Fungemia at a tertiary care hospital: Incidence, therapy, and distribution and antifungal susceptibility of causative species," European Journal of Clinical Microbiology and Infectious Diseases, vol. 26, no. 8, pp. 541-547, 2007.

[12] E. Sarvikivi, O. Lyytikäinen, D. R. Soll et al., "Emergence of fluconazole resistance in a Candida parapsilosis strain that caused infections in a neonatal intensive care unit," Journal of Clinical Microbiology, vol. 43, no. 6, pp. 2729-2735, 2005.

[13] A. Spiliopoulou, G. Dimitriou, E. Jelastopulu, I. Giannakopoulos, E. D. Anastassiou, and M. Christofidou, "Neonatal Intensive Care Unit Candidemia: Epidemiology, Risk Factors, Outcome, and Critical Review of Published Case Series," Mycopathologia, vol. 173, no. 4, pp. 219-228, 2012.

[14] G. Lovero, O. De Giglio, O. Montagna et al., "Epidemiology of candidemia in neonatal intensive care units: A persistent public health problem," Annali di Igiene, vol. 28, no. 4, pp. 282-287, 2016.

[15] M. T. Montagna, G. Lovero, O. De Giglio, R. Iatta, G. Caggiano, O. Montagna et al., Journal of Preventive Medicine and Hygiene, vol. 51, 130, 125, 2010.

[16] A. M. Tortorano, A. Prigitano, C. Lazzarini et al., "A 1-year prospective survey of candidemia in Italy and changing epidemiology over one decade," Infection, vol. 41, no. 3, pp. 655662, 2013.

[17] D. Rodriguez, B. Almirante, B. J. Park et al., "Candidemia in neonatal intensive care units: Barcelona, Spain," Pediatric Infectious Disease Journal, vol. 25, no. 3, pp. 224-229, 2006.

[18] J. Pemán, E. Cantón, M. J. Linares-Sicilia et al., "Epidemiology and antifungal susceptibility of bloodstream fungal isolates in pediatric patients: A Spanish multicenter prospective survey," Journal of Clinical Microbiology, vol. 49, no. 12, pp. 4158-4163, 2011.

[19] M. Yalaz, H. Çetin, M. Akisu, Ş. Aydemir, A. Tunger, and N. Kültürsay, "Neonatal nosocomial sepsis in a level-III NICU: Evaluation of the causative agents and antimicrobial susceptibilities," Turkish Journal of Pediatrics, vol. 48, no. 1, pp. 13-18, 2006.

[20] S. Celebi, M. Hacimustafaoglu, N. Koksal, H. Ozkan, M. Cetinkaya, and B. Ener, "Neonatal candidiasis: Results of an 8 year study," Pediatrics International, vol. 54, no. 3, pp. 341-349, 2012.

[21] H. Ozkan, M. Cetinkaya, N. Koksal, S. Celebi, and M. Hacimustafaoglu, "Culture-proven neonatal sepsis in preterm infants in a neonatal intensive care unit over a 7 year period: coagulasenegative Staphylococcus as the predominant pathogen," Pediatrics International, vol. 56, no. 1, pp. 60-66, 2014.

[22] L. Clerihew, T. L. Lamagni, P. Brocklehurst, and W. McGuire, "Invasive fungal infection in very low birthweight infants: National prospective surveillance study," Archives of Disease in Childhood: Fetal and Neonatal Edition, vol. 91, no. 3, pp. F188F192, 2006.

[23] S. Vergnano, E. Menson, N. Kennea et al., "Neonatal infections in England: The neonIN surveillance network," Archives of Disease in Childhood: Fetal and Neonatal Edition, vol. 96, no. 1, pp. F9-F14, 2011.

[24] M. Aziz, A. L. Patel, J. Losavio et al., "Efficacy of fluconazole prophylaxis for prevention of invasive fungal infection in extremely low birth weight infants," Pediatric Infectious Disease Journal, vol. 29, no. 4, pp. 352-356, 2010. 
[25] K. N. Feja, F. Wu, K. Roberts et al., "Risk factors for candidemia in critically ill infants: a matched case-control study," Journal of Pediatrics, vol. 147, no. 2, pp. 156-161, 2005.

[26] D. L. Horn, D. Neofytos, E. J. Anaissie et al., "Epidemiology and outcomes of candidemia in 2019 patients: data from the prospective antifungal therapy alliance registry," Clinical Infectious Diseases, vol. 48, no. 12, pp. 1695-1703, 2009.

[27] M. Pfaller, D. Neofytos, D. Diekema et al., "Epidemiology and outcomes of candidemia in 3648 patients: data from the Prospective Antifungal Therapy (PATH Alliance) registry, 2004-2008," Diagnostic Microbiology and Infectious Disease, vol. 74, no. 4, pp. 323-331, 2012.

[28] M. J. Bizzarro, V. Shabanova, R. S. Baltimore, L.-M. Dembry, R. A. Ehrenkranz, and P. G. Gallagher, "Neonatal sepsis 2004-2013: The rise and fall of coagulase-negative staphylococci," Journal of Pediatrics, vol. 166, no. 5, pp. 1193-1199, 2015.

[29] G. Natarajan, M. Lulic-Botica, and J. V. Aranda, "Refractory neonatal candidemia and high-dose micafungin pharmacotherapy," Journal of Perinatology, vol. 29, no. 11, pp. 738-743, 2009.

[30] J. A. Robinson, H. D. Pham, B. T. Bloom, and R. R. Wittler, "Risk factors for persistent candidemia infection in a neonatal intensive care unit and its effect on mortality and length of hospitalization," Journal of Perinatology, vol. 32, no. 8, pp. 621625, 2012.

[31] G. C. M. Batista, V. L. J. Krebs, L. S. Ruiz, M. E. Auler, R. C. Hahn, and C. R. Paula, "Oral colonization: A possible source for candidemia in low-weight neonates," Journal de Mycologie Medicale, vol. 24, no. 2, pp. 81-86, 2014.

[32] H. D. Hoffmann-Santos, C. R. Paula, A. C. A. Yamamoto, T. Tadano, and R. C. Hahn, "Six-Year Trend Analysis of Nosocomial Candidemia and Risk factors in Two Intensive Care Hospitals in Mato Grosso, Midwest Region of Brazil," Mycopathologia, vol. 176, no. 5-6, pp. 409-415, 2013.

[33] J. A. Cortés, P. Reyes, C. Gómez, G. Buitrago, and A. L. Leal, "Fungal bloodstream infections in tertiary care hospitals in Colombia," Revista Iberoamericana de Micología, vol. 28, no. 2, pp. 74-78, 2011.

[34] J. A. Cortés, P. Reyes, C. H. Gómez et al., "Clinical and epidemiological characteristics and risk factors for mortality in patients with candidemia in hospitals from Bogotá, Colombia," Brazilian Journal of Infectious Diseases, vol. 18, no. 6, pp. 631-637, 2014.

[35] S. Hua, J. Huang, Z. Wu, and Z. Feng, "A comparison study between Candida parapsilosis sepsis and Candida albicans sepsis in preterm infants," Turkish Journal of Pediatrics, vol. 54, no. 5, pp. 502-508, 2012.

[36] Z. Wu, Y. Liu, X. Feng et al., "Candidemia: Incidence rates, type of species, and risk factors at a tertiary care academic hospital in China," International Journal of Infectious Diseases, vol. 22, pp. e4-e8, 2014.

[37] I. Chen, N. Chiu, H. Chi et al., "Changing of bloodstream infections in a medical center neonatal intensive care unit," Journal of Microbiology, Immunology and Infection, 2015.

[38] R. Rani, N. P. Mohapatra, G. Mehta, and V. S. Randhawa, "Changing trends of Candida species in neonatal septicaemia in a tertiary North Indian hospital," Indian Journal of Medical Microbiology, pp. 2042-2044, 2002.

[39] J. Agarwal, S. Bansal, G. K. Malik, and A. Jain, "Trends in neonatal septicemia: Emergence of non-albicans Candida," Indian Pediatrics, vol. 41, no. 7, pp. 712-715, 2004.

[40] P. Femitha, R. Joy, B. Adhisivam et al., "Candidemia in neonatal ICU- experience from a tertiary care hospital," Current Pediatric Research, vol. 17, no. 1, pp. 44-48, 2013.
[41] V. Mehara, D. Yadava, P. Somania, G. Bhatambareb, S. Mulyea, and K. Singh, "Neonatal sepsis in a tertiary care center in central India: microbiological profile, antimicrobial sensitivity pattern and outcome," Journal of Neonatal-Perinatal Medicine, vol. 6, pp. 165-172, 2013.

[42] D. Juyal, M. Sharma, S. Pal, V. K. Rathaur, and N. Sharma, "Emergence of non-albicans Candida species in neonatal candidemia," North American Journal of Medical Sciences, vol. 5, no. 9, pp. 541-545, 2013.

[43] D. Chaurasia, M. Goel, S. Dhruw, D. Dubey, and R. Dwivedi, "Changing Pattern of Neonatal Fungal Sepsis: A Matched Case Control Study," National Journal of Medical and Allied Sciences, vol. 4, no. 1, 2015, Online first.

[44] R. Wadile and V. Bhate, "Study of clinical spectrum and risk factors of neonatal candidemia," Indian Journal of Pathology and Microbiology, vol. 58, no. 4, pp. 472-474, 2015.

[45] N. Al-Sweih, Z. Khan, S. Khan, and L. V. Devarajan, "Neonatal candidaemia in Kuwait: A 12-year study of risk factors, species spectrum and antifungal susceptibility," Mycoses, vol. 52, no. 6, pp. 518-523, 2009.

[46] M. S. Hammoud, A. Al-Taiar, M. Fouad, A. Raina, and Z. Khan, "Persistent candidemia in neonatal care units: Risk factors and clinical significance," International Journal of Infectious Diseases, vol. 17, no. 8, pp. e624-e628, 2013.

[47] E. A. Khan, S. Choudhry, M. Fatima, and Z. Batool, "Clinical spectrum, management and outcome of neonatal candidiasis," Journal of the Pakistan Medical Association, vol. 65, pp. 12061209, 2015.

[48] J.-H. Wu, C.-Y. Chen, P.-N. Tsao, W.-S. Hsieh, and H.-C. Chou, "Neonatal sepsis: a 6-year analysis in a neonatal care unit in Taiwan," Pediatrics \& Neonatology, vol. 50, no. 3, pp. 88-95, 2009.

[49] M.-H. Tsai, J.-F. Hsu, S.-M. Chu et al., "Incidence, clinical characteristics and risk factors for adverse outcome in neonates with late-onset sepsis," Pediatric Infectious Disease Journal, vol. 33, no. 1, pp. e7-e13, 2014.

[50] W. H. Lim, R. Lien, Y.-C. Huang et al., "Prevalence and pathogen distribution of neonatal sepsis among very-low-birth-weight infants," Pediatrics and Neonatology, vol. 53, no. 4, pp. 228-234, 2012.

[51] D. E. Ballot, N. Bosman, T. Nana, T. Ramdin, and P. A. Cooper, "Background changing patterns of neonatal fungal sepsis in a developing country," Journal of Tropical Pediatrics, vol. 59, no. 6, Article ID fmt053, pp. 460-464, 2013.

[52] S. Chen, M. Slavin, Q. Nguyen et al., "Active Surveillance of Candidemia, Australia," Emerging Infectious Diseases, vol. 12, no. 10, pp. 1508-1516, 2006.

[53] L. Clerihew, T. L. Lamagni, P. Brocklehurst, and W. McGuire, "Candida parapsilosis infection in very low birthweight infants," Archives of Disease in Childhood: Fetal and Neonatal Edition, vol. 92, no. 2, pp. F127-F129, 2007.

[54] S.-K. Kim, K. El Bissati, and C. B. Mamoun, "Amino acids mediate colony and cell differentiation in the fungal pathogen Candida parapsilosis," Microbiology, vol. 152, no. 10, pp. 28852894, 2006.

[55] A. A. Lattif, P. K. Mukherjee, J. Chandra et al., "Characterization of biofilms formed by Candida parapsilosis, C. metapsilosis, and C. orthopsilosis," International Journal of Medical Microbiology, vol. 300, no. 4, pp. 265-270, 2010.

[56] P. G. Pappas, C. A. Kauffman, D. R. Andes, C. J. Clancy, K. A. Marr, L. Ostrosky-Zeichner et al., "Clinical practice guideline 
for the management of candidiasis: 2016 update by the infectious diseases society of America," Clinical Infectious Diseases, vol. 62, pp. e1-50, 2016.

[57] L. P. Brion, S. E. Uko, and D. L. Goldman, "Risk of resistance associated with fluconazole prophylaxis: Systematic review," Journal of Infection, vol. 54, no. 6, pp. 521-529, 2007. 


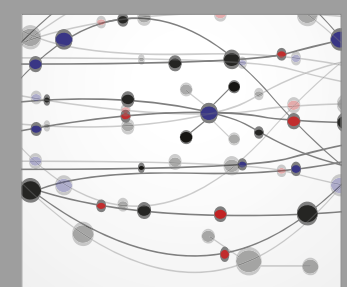

The Scientific World Journal
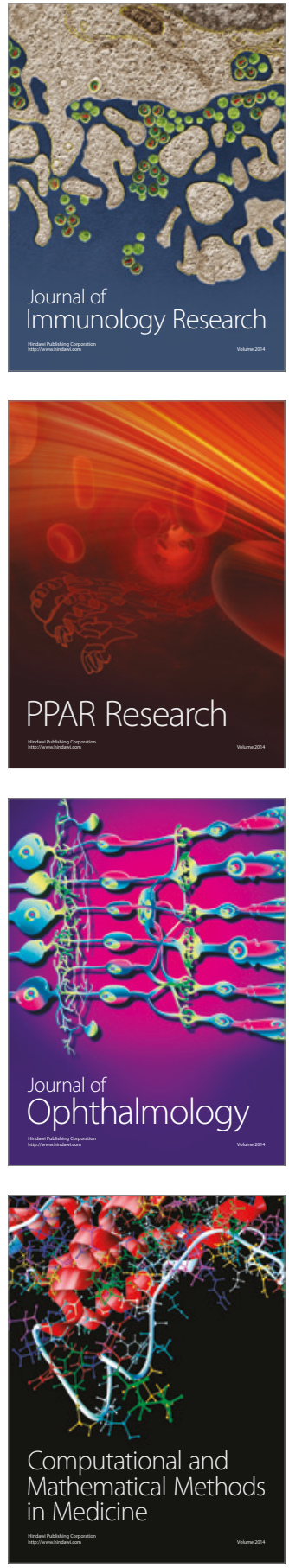

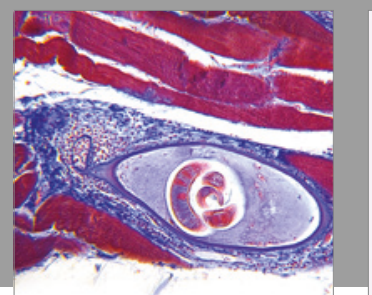

Gastroenterology Research and Practice
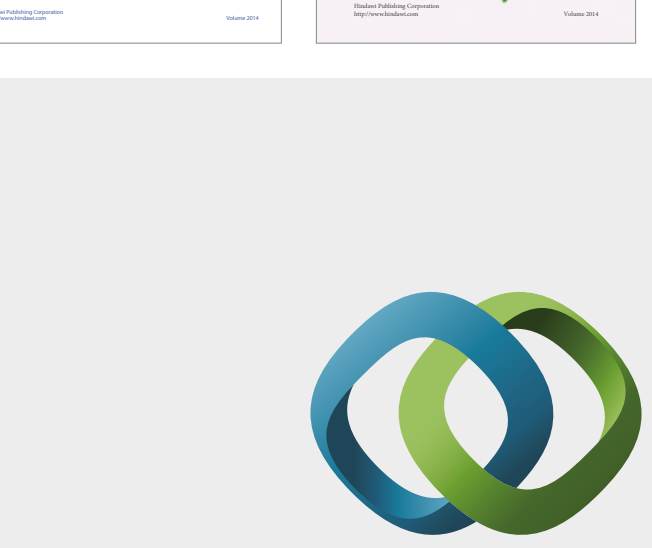

\section{Hindawi}

Submit your manuscripts at

https://www.hindawi.com
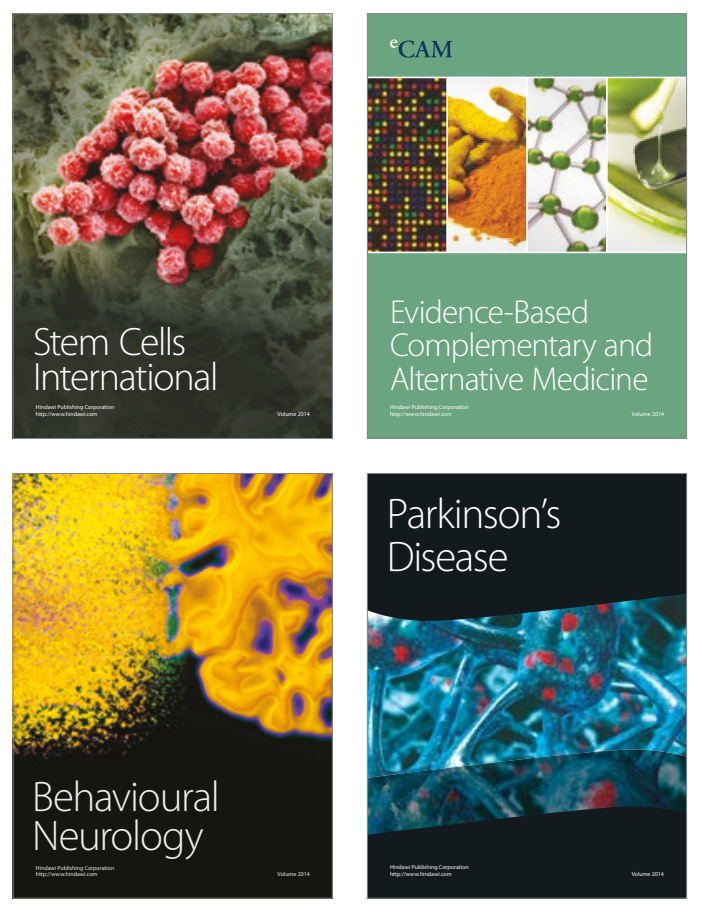
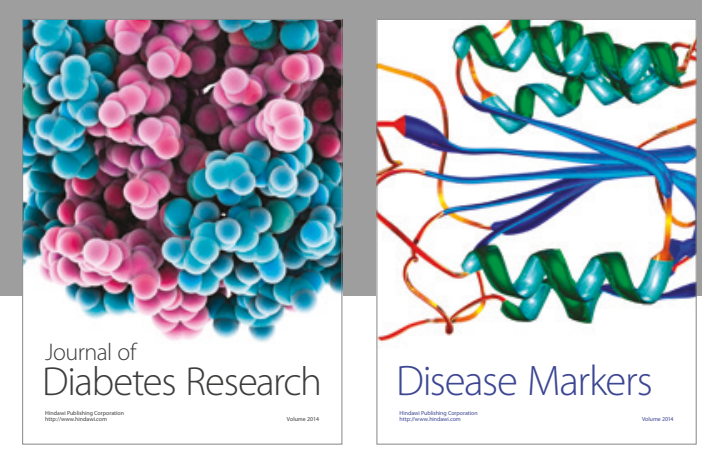

Disease Markers
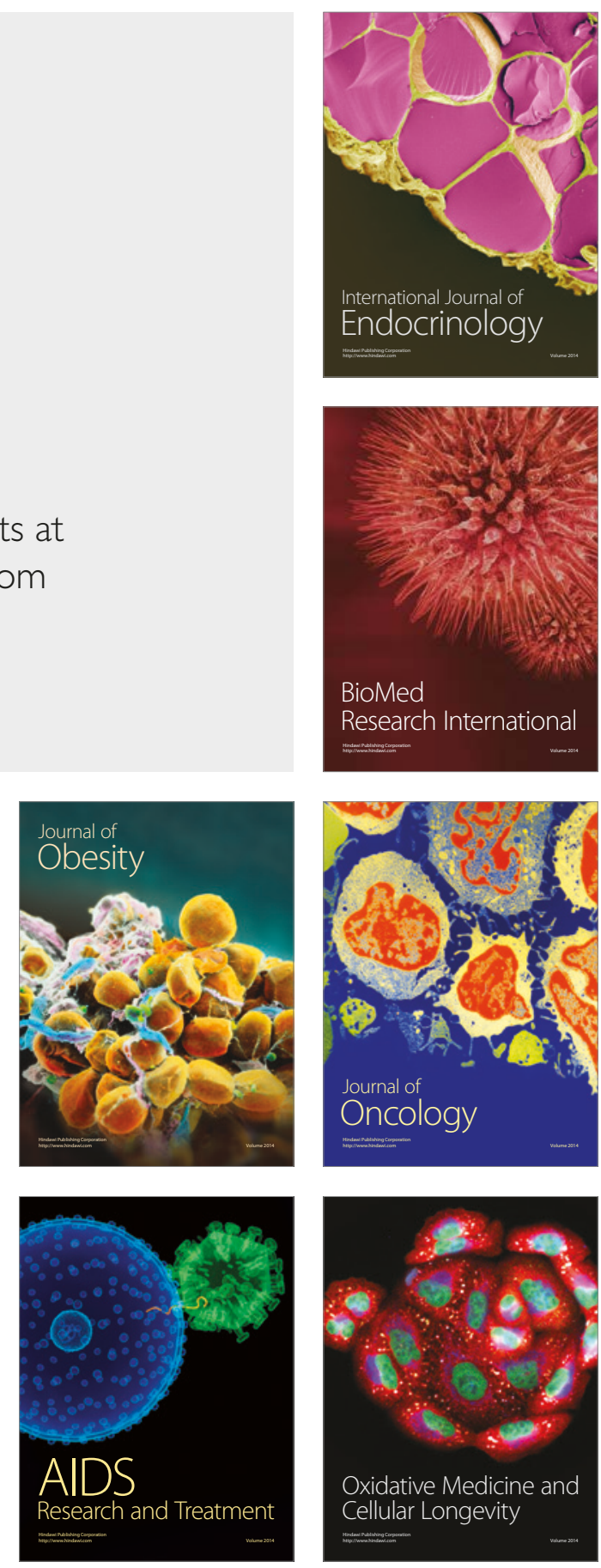